\title{
Celastrol Modulates Multiple Signaling Pathways to Inhibit Proliferation of Pancreatic Cancer via DDIT3 and ATF3 Up-Regulation and RRM2 and MCM4 Down-Regulation
}

\author{
Mahmoud Youns (iD) ${ }^{1,2}$ \\ Momen Askoura (iD ${ }^{3}$ \\ Hisham A Abbas (D) ${ }^{3}$ \\ Gouda H Attia ${ }^{4,5}$ \\ Ahdab N Khayyat ${ }^{6}$ \\ Reham M Goda ${ }^{7}$ \\ Ahmad J Almalki ${ }^{6}$ \\ El-Sayed Khafagy ${ }^{8,9}$ \\ Wael AH Hegazy (iD ${ }^{3}$ \\ 'Department of Biochemistry and \\ Molecular Biology, Faculty of Pharmacy, \\ Helwan University, Cairo, Egypt; \\ ${ }^{2}$ Department of Functional Genome \\ Analysis, German Cancer Research \\ Center (DKFZ), Heidelberg, 69I20, \\ Germany; ${ }^{3}$ Department of Microbiology \\ and Immunology, Faculty of Pharmacy, \\ Zagazig University, Zagazig, Egypt; \\ ${ }^{4}$ Department of Pharmacognosy, Faculty \\ of Pharmacy, Kafr El-Shiekh University, \\ Kafr El-Shiekh, Egypt; ${ }^{5}$ Department of \\ Pharmacognosy, College of Pharmacy, \\ Najran University, Najran, Saudi Arabia; \\ ${ }^{6}$ Department of Pharmaceutical \\ Chemistry, Faculty of Pharmacy, King \\ Abdulaziz University, Jeddah, 21589. \\ Saudi Arabia; ${ }^{7}$ Department of \\ Microbiology and Biotechnology, Faculty \\ of Pharmacy, Delta University for Science \\ and Technology, Gamasa, Mansoura, \\ Egypt; ${ }^{8}$ Department of Pharmaceutics, \\ College of Pharmacy, Prince Sattam Bin \\ Abdulaziz University, Al-kharj, I 1942, \\ Saudi Arabia; ${ }^{9}$ Department of \\ Pharmaceutics and Industrial Pharmacy, \\ Faculty of Pharmacy, Suez Canal \\ University, Ismailia, 4I552, Egypt
}

Correspondence: Mahmoud Youns Tel +0096891742704

Email dr.mahmoudyouns@yahoo.com
Background: Pancreatic cancer is one of the most serious and lethal human cancers with a snowballing incidence around the world. The natural product celastrol has also been widely documented as a potent anti-inflammatory, anti-angiogenic, and anti-oxidant.

Purpose: To elucidate the antitumor effect of celastrol on pancreatic cancer cells and its modulatory role on whole genome expression.

Methods: The antitumor activity of celastrol on a panel of pancreatic cancer cells has been evaluated by Sulforhodamine B assay. Caspase 3/7 and histone-associated DNA fragments assays were done for apoptosis measurement. Additionally, prostaglandin (PGE2) inhibition was evaluated. Moreover, a microarray gene expression profiling was carried out to detect possible key players that modulate the antitumor effects of celastrol on cells of pancreatic cancer. Results: Our findings indicated that celastrol suppresses the cellular growth of pancreatic cancer cells, induces apoptosis, and inhibits PGE2 production. Celastrol modulated many signaling genes and its cytotoxic effect was mainly mediated via over-expression of $A T F 3$ and DDIT3, and down-expression of RRM2 and MCM4.

Conclusion: The current study aims to be a starting point to generate a hypothesis on the most significant regulatory genes and for a full dissection of the celastrol possible effects on each single gene to prevent the pancreatic cancer growth.

Keywords: celastrol, natural products, pancreatic cancer, RRM2, DDIT3, MCM4

\section{Introduction}

Pancreatic cancer is ranked among the top five death-related-malignancies in the United States and accounts for over 43,000 deaths annually and an estimated 53,000 new diagnosed cases. ${ }^{1,2}$ The survival rate still remains one of the lowest among other types of malignant diseases, with $5 \%$ of patients living over 5 years. ${ }^{1-5}$ Early diagnosis of the tumor is considered the best tool to combat the disease. However, in practice, this is considered as an unrealistic option for several reasons. Surgical intervention, on the other hand, is the best treatment option so far for pancreatic cancer patients, despite its low survival rates $(20 \%)$. Moreover, it has been estimated that nearly $80 \%$ of the patients do not undergo surgical resection due to the difficulties in early diagnosis. Alternatively, chemotherapy or chemoradiotherapy is still an available therapeutic option, in particular for patients who suffer from local unresectable or advanced metastatic tumors. ${ }^{6}$ 


\section{Graphical Abstract}

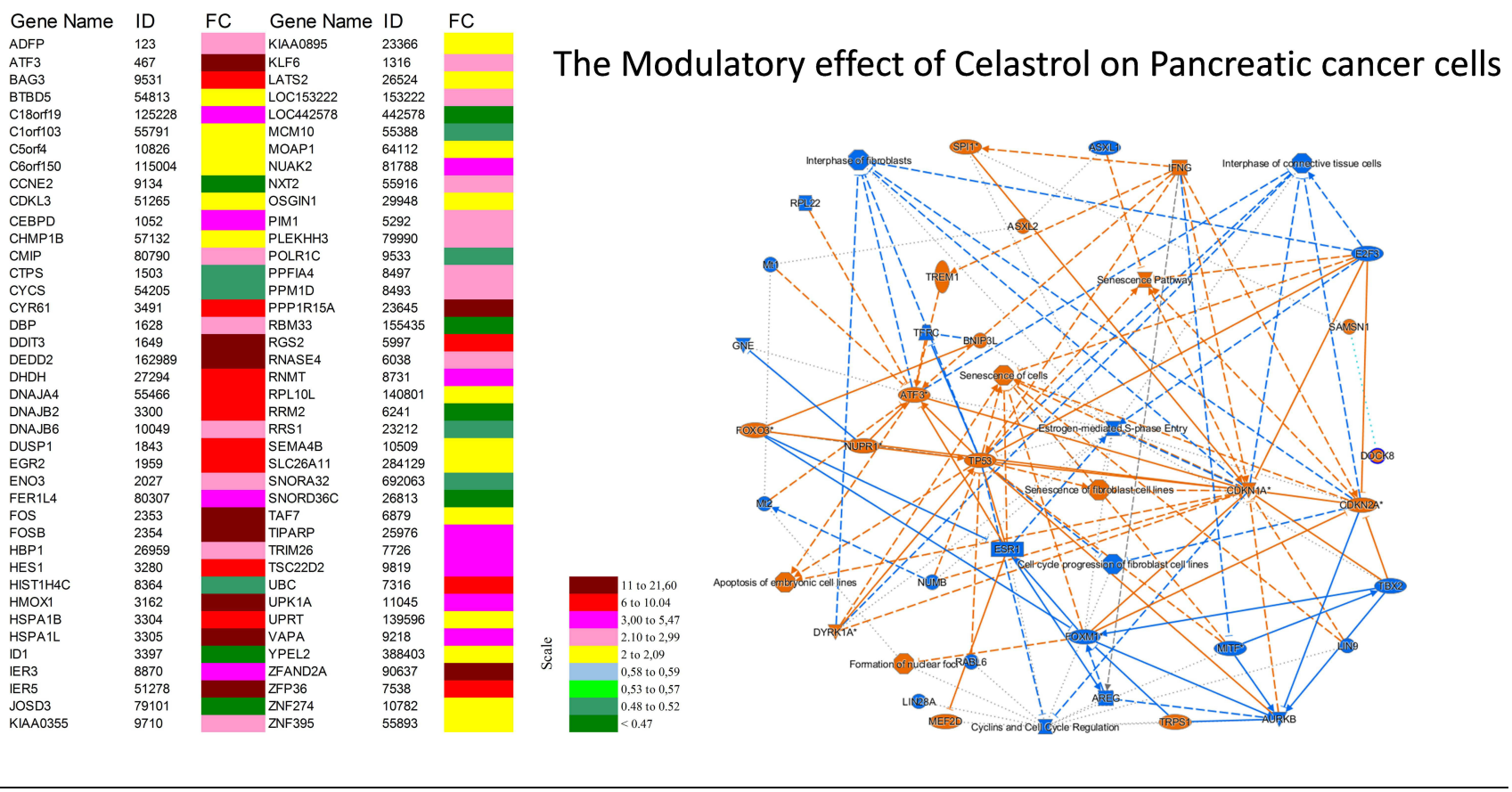

The use of natural products and their derivatives has always rising attention to mitigate the seriousness of various diseases including cancers; that is gripping the attention of search groups worldwide. ${ }^{7-13}$ Recently, the immense bioactivities of natural products have been pinpointed; that they can modulate the signaling pathways in tumor cells via activation or suppression of multiple cellular pathways. ${ }^{3,8,14-18}$ Celastrol, a quinone methide triterpenoid, is a natural product extracted from Tripterygium wilfordii Hook F. ("Thunder of God Vine") plant and is used widely in TCM (traditional Chinese medications). The biological activities of celastrol have been extensively explored; it has been documented that celastrol has anticancer activity against various tumors, including leukemia, melanoma glioma, and prostate cancer. ${ }^{19-28}$ The celastrol anticancer activity was owed to its capability to compromise different cellular mechanisms in cancer cells. Celastrol is found to suppress tumor necrotic factor (TNF) induced cancer cells metastasis, nuclear factor (NF)-kB signaling, heat shock protein (HSP) 90, extracellular signal-regulated kinase (ERK), proteasome, and modulates TRAIL-induced apoptosis. ${ }^{29-35}$

To our knowledge, the antitumor activity of celastrol on pancreatic cancer in different proliferation stages and its influence on the expression pattern of the whole genome is not yet identified. In the current work, we aimed to evaluate the inhibitory effects of celastrol on the growth of human pancreatic malignant cells at different stages. In this context, we investigated the antitumor activity of celastrol on numerous cell lines representing drug resistant patterns and diverse differentiation stages of pancreatic cancer in comparison to normal immortalized pancreatic cells.

\section{Materials and Methods}

\section{Pancreatic Cell Lines}

Eight pancreatic human cancer cell lines were obtained from the American Type Culture Collection (Rockville, USA) and were used in this study: Suit-007, Suit-2, BxPC-3, Capan-2, Capan-1, Miapaca-2, Colo-357, and Panc-1 as well as the normal immortalized pancreatic cell line HPDE. Cell lines were grown and treated with celastrol or control dimethyl sulfoxide (DMSO) as previously indicated. $5,8,13,16,18,36-39$

\section{Effect of Celastrol on Cellular Proliferation}

To evaluate the anti-proliferative effects of celastrol on cells of pancreatic cancer, the Sulforhodamine B (SRB) assay was accomplished to evaluate the cellular protein 
content. $^{4,5,9,10,13,17}$ Briefly, cell lines were incubated and gradually treated for 48 hours with DMSO or increasing doses of celastrol. Then, cells were fixed with trichloroacetic acid (TCA) (10\%) and stained with SRB fluorescent dye for 30 minutes. The excess dye was repeatedly washed with $1 \%$ acetic acid and the SRB bounded to cellular proteins was then dissolved in $10 \mathrm{mM}$ Tris base. The absorbance was measured at $510 \mathrm{~nm}$ in a reader (Molecular Devices, Sunnyvale, CA, USA).

\section{Evaluation of the Apoptotic Effects of Celastrol on Pancreatic Cancer Cell Lines}

The apoptotic ability of celastrol was determined by measuring degradation of DNA and histone-associated DNA fragments. The apoptotic effects of celastrol on BxPC-3, Panc-1, and Miapaca-2 cancer cells were evaluated using the ELISA kit for detection of cell death (Roche Diagnostics $\mathrm{GmbH}$, Germany). Briefly, cells were either dosed with or without celastrol ( $1 \frac{2}{2}$ IC50, IC 50 , or 2 IC50) for 6 hours. The cytoplasm of untreated and treated cells was then transferred to coated plates with Biotin-binding protein streptavidin. The plates were incubated with biotinylated histone antibody and peroxidase-tagged mouse anti-human DNA, and the absorbance was measured at $405 \mathrm{~nm}$ (Molecular Devices, Sunnyvale, CA, USA).

\section{Evaluation of Caspase-3/7 Activity}

Caspases are protease enzymes that play crucial roles in apoptosis. In this context, the apoptotic effects of celastrol on pancreatic cell line Panc-1 were further confirmed through measuring caspase 3/7 quantities using assay kit (Caspase-Glo 3/7) (Promega, Fitchburg, MA, USA) as previously performed. ${ }^{8,9,18,37,40-42}$ Briefly, cells were dosed with or without celastrol ( $1 / 2$ IC50, IC50, or 2 IC50) for 6 hours. Reagent Caspase-Glo 3/7 was prepared, added in equal volumes to cells, mixed gently, and incubated for 1 hour at $25^{\circ} \mathrm{C}$. The luminescence was detected, and the activity of caspase was presented as a percentage of the untreated control.

\section{Inhibition Assay of Prostaglandin}

To evaluate the celastrol's effect on the production of prostaglandin E2 (PGE2), the prostaglandin E2 assay Kit (Cayman Chemicals, MI, USA) was employed as indicated formerly. $3,8,38,43$ The assay is designed to detect and quantify the level of human PGE2 in biological samples. It is based on the competition between PGE2 and a PGE2 alkaline phosphatase tracer for a restricted amount of specific PGE2 monoclonal antibody. Furthermore, NS398, non-steroidal anti-inflammatory, was employed as a positive control. Briefly, after treatment, cells were dosed with or without celastrol ( $1 / 2$ IC50, IC50, or 2 IC50) for 24 hours and stimulated with arachidonic acid. The PGE2 production was assessed by a competitive enzyme immunoassay in accordance with the kit manufacturer's procedure. The inhibitory effects of celastrol and positive control were presented as a percentage of the untreated control.

\section{Microarray}

In order to perform a whole genome expression analysis, the high COX-2 expressing BxPC-3 cells were either dosed with $10 \mu \mathrm{M}$ celastrol or DMSO for 2 days. RNA was extracted, purified, and processed as we described previously. ${ }^{37-39,41}$ The Illumine human sentrix- 8 v3. The Human sentrix Ref-8 Expression BeadChip contains eight arrays of more than 24,000 probes derived from the RefSeq database. These new Expression BeadChips provide high-quality performance with low rates of false differential expression. Here, the differential expression values are defined as genes showing a more than or equal to 1.5-fold difference in raw hybridization intensity (up or down) between two samples.

\section{Validation of Microarray by RT-PCR}

The significance of array results has been checked and validated by RT-PCR analysis for selected significantly regulated genes throughout the array study. The RT-PCR was performed with the same samples used for microarray according to QuantiTect SYBR Green Kit (Qiagen, Germany). ${ }^{37,41,44,45}$

\section{Determination of the Tangled Effects of Celastrol on Signaling Pathways in Cells of Pancreatic Cancer}

The Ingenuity Pathway Analysis (IPA) (www.ingenuity. com) (Mountain View, USA) was employed in our study to identify the expected molecular biomarkers list, networks and signaling pathways that have been modified after treatment of celastrol, as previously shown. ${ }^{3,5,8,16,36}$

\section{Data Analysis and Statistical Analysis}

Microarray data analysis, cluster analysis, normalization, and quality assessment were carried out with the MIAME 
compatible analysis where differences with a $P$-value $<0.05$ were only considered. Moreover, correspondence analysis was employed for cluster analysis. ${ }^{5,37,39,41}$ The assays were conducted in triplicate and the results are presented as the means \pm standard errors and $P$-values $<0.05$ were assumed to be statistically significant using two-way ANOVA test (Graphpad Prism 8 software).

\section{Results}

\section{Celastrol Suppresses Cellular} Proliferation of Cells of Pancreatic

\section{Cancer}

The celastrol effects on cellular proliferation of eight different pancreatic cancer cell lines (Suit-2, Suit-007, BxPC-3, Capan-2, Colo-357, Miapaca-2, Panc-1, and Capan-1) and the normal immortalized pancreatic cells (HPDE) have been evaluated. SRB colorimetric assay has been used to evaluate the cellular proliferation with steady rising in celastrol dosing for 48 hours. Results showed that Miapaca-2 and BxPc-3 were the most affected cells (IC50=7.31 \pm 0.33 and $7.79 \pm 0.81$, respectively), while Capan-2 and Panc-1 cells were the least affected cancer ones (IC50 $=11.46 \pm 0.83$ and $16.15 \pm 0.97$, respectively). Meanwhile, the normal HPDE cells were the least affected after treatment (IC50>20 $\mu \mathrm{M})$ (Table 1).

\section{Celastrol Induces Apoptotic Cell Death}

Induction of apoptotic cascade is a main chemotherapyinduced cell death mechanism. ${ }^{46}$ The apoptotic effects of

Table I Celastrol Inhibits Proliferation of Pancreatic Cancer Cell Lines. Eight Different Pancreatic Cancer Cell Lines Were Treated with Increasing Concentrations of Celastrol and IC50 Values Have Been Accordingly Calculated

\begin{tabular}{|l|c|}
\hline Cell Line & IC $_{50}(\mu \mathrm{M})$ \\
\hline Miapaca-2 cells & $7.3 I \pm 0.33$ \\
\hline Capan-I cells & $8.24 \pm 0.54$ \\
\hline Capan-2 cells & $I I .46 \pm 0.83$ \\
\hline Suit-007 cells & $8.64 \pm 0.7 I$ \\
\hline BxPC-3 cells & $7.79 \pm 0.8 I$ \\
\hline Colo-357 cells & $8.76 \pm 0.56$ \\
\hline Panc-I cells & $16.15 \pm 0.97$ \\
\hline Suit-2 cells & $10.38 \pm 0.92$ \\
\hline HPDE cells & $>20$ \\
\hline
\end{tabular}

celastrol on pancreatic resistant cell lines Miapaca-2, BxPC-3, and Panc-1 were evaluated by measurement of histone linked DNA fragments in cytosol and caspase 3/7 levels. Results revealed that celastrol provoked apoptosis via increasing the number of fragmented DNA released in cytoplasm (Figure 1) and, additionally, by increasing the amount of activated caspases 3/7 (Figure 2) in Panc-1, BxPC-3, and $\mathrm{MiaPaCa}-2$ cell lines compared to untreated controls. The apoptotic effect was dose-dependent in all tested cell lines.

\section{Celastrol Prevents PGE2 Production}

To appraise celastrol anti-inflammatory effect via PGE2 inhibition, we hired the high COX expression cell line (BxPC-3). Our findings indicated that celastrol considerably decreased PGE2 production openly with dose increasing confirming the anti-inflammatory effect of celastrol on pancreatic cancer cells and the possible beneficial effect for cancer treatment (Figure 3).

\section{Target Genes and Signaling Pathways After BxPc-3 Cells Treatment with Celastrol}

Microarray expression testing has been conceded as formerly designated $^{37,39,41}$ using Illumine human sentrix-8 v3 (Chip 5388892088). For significance a threshold of typically 0.05 is chosen: Reject all as not significant, where $p \geq 0.05$. In our study, BxPC-3 cells were dosed with DMSO as control or 10 $\mu \mathrm{M}$ celastrol for 48 hours. Gene regulation outcomes indicated that celastrol drastically regulated 300 genes in BxPc-3 cells, with a minimum of 2-folds fluctuation in their expression. Two hundred and eight and 92 genes were significantly up- or down-regulated, respectively. The top 80 regulated genes are shown (Figure 4). The color-coded scale shows fold changes for upregulated and down-regulated genes. Regulated genes were primarily implicated in cyclin and cell cycle regulation. Importantly, array results agreed with RT-PCR results (Table 2) where both the DNA-damage-inducible transcript 4 (DDIT4), the Growth Arrest and DNA Damage Inducible Alpha (GADD45B) and Fos Proto-Oncogene (Fos) were commonly upregulated and the Ribonucleotide Reductase Regulatory Subunit M2 (RRM2) was commonly downregulated in both array and RT-PCR results after treatment.

\section{Analysis of Canonical and Signaling Pathways}

To recognize the canonical pathways and networks facilitating the celastrol inhibitory effects on the BxPC-3 


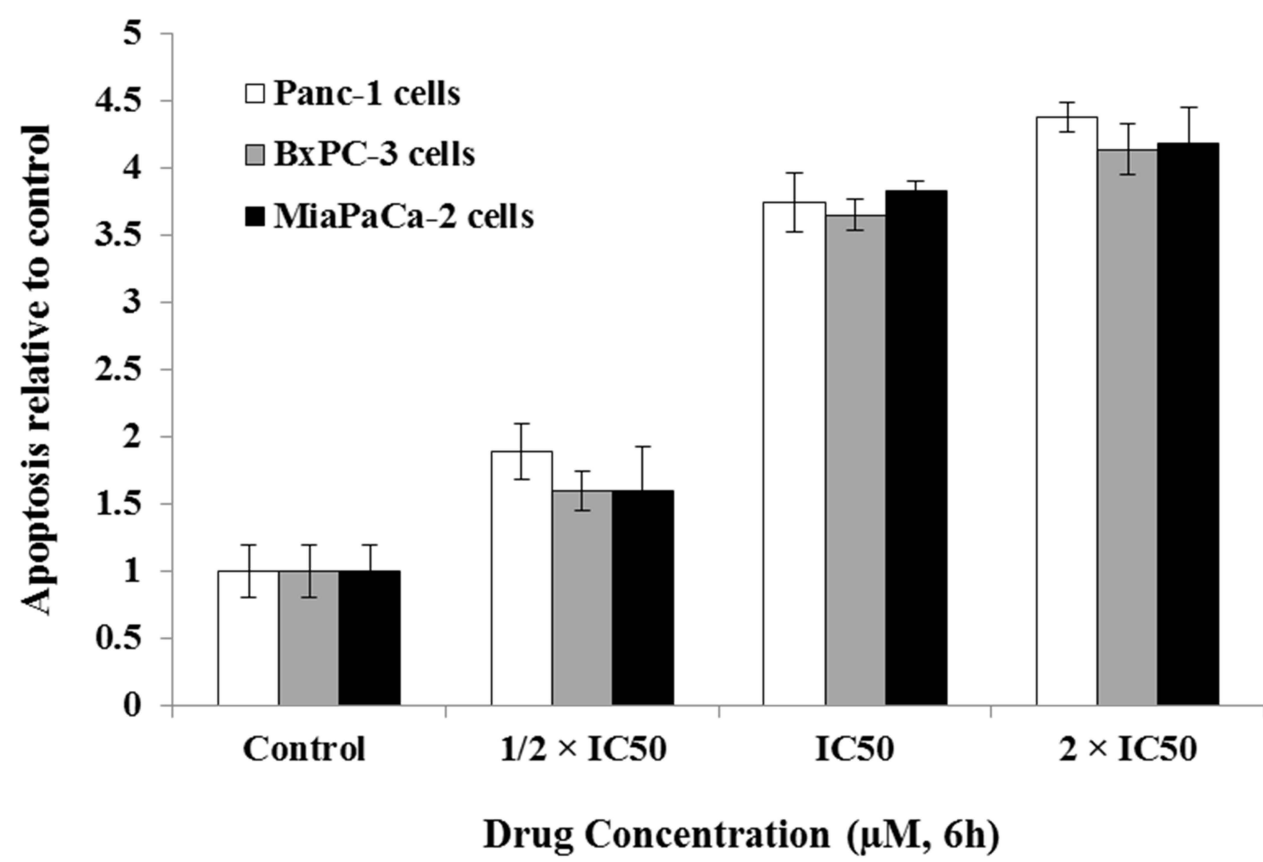

Figure I Celastrol induced apoptosis of cancer cell. Cell lines were treated with different concentrations of celastrol and histone-associated DNA fragments were quantified by SRB assay. The assay was repeated in triplicate and the results are shown as means \pm SEM.

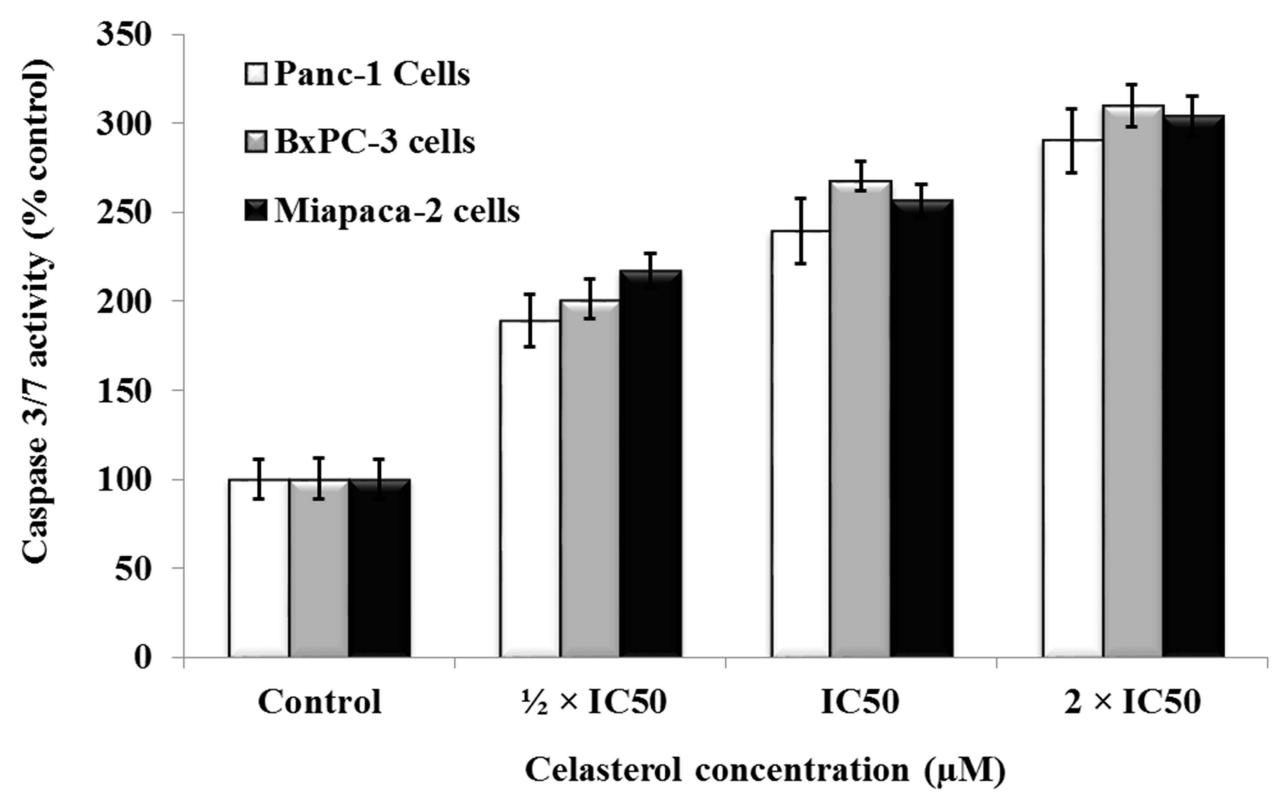

Figure 2 Celastrol increases caspases. Cell lines were treated with different concentrations of celastrol and caspases $3 / 7$ assay was performed. The assay was repeated three times and the results were presented as means \pm SEM.

cancer cell line, the IPA was employed. ${ }^{37}$ The IPA Graphical Summary gives an outline of the key biological themes in our IPA core analysis and explains how these thoughts communicate with each other, creating a coherent and comprehensible synopsis of the analysis. The topmost five regulated canonical pathways postcelastrol treatment were cyclins and cell cycle regulation
(Figure 5), senescence pathway, cell cycle control and chromosomal regulation, cell cycle: G1/S checkpoint regulation (Figure 6), apoptosis of embryonic cell lines, estrogen-mediated S-phase entry, and prostatic cancer signaling. Figure 6 shows the number of upregulated genes (red) involved in cell cycle: G1/S checkpoint regulation like $P 27 K 1 P 1$, and $P 18 I N K 4 C$ and additionally 


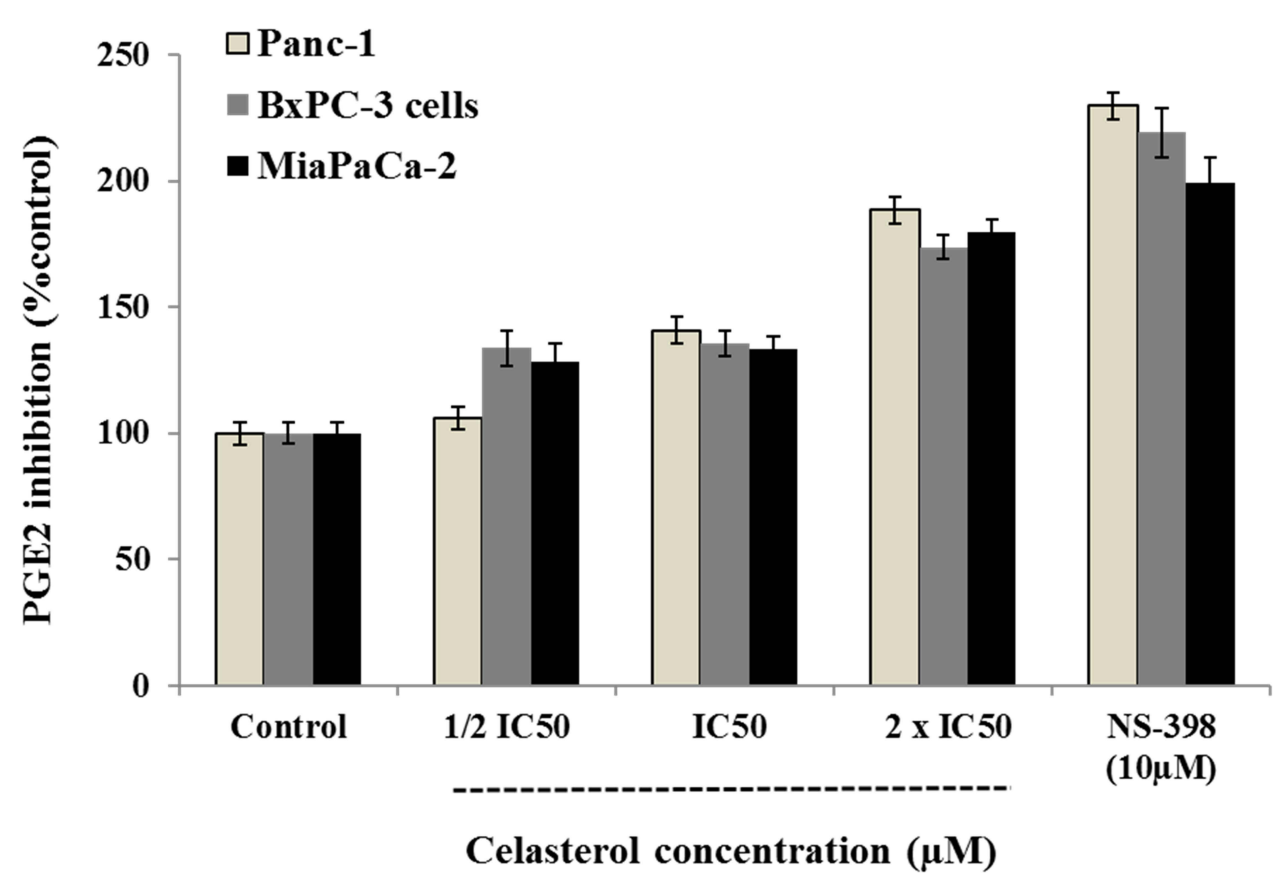

Figure 3 Celastrol inhibits PGE2 production in BxPC-3 cells. BxPC-3 cells were treated with different concentrations of celastrol and PGE2 production was assessed. The assay was conducted three times and the results were expressed as means \pm SEM.

the number of downregulated genes (green) involved in cell cycle: $G 1 / S$ checkpoint regulation like $C y c l i n B$, Cyclin A, and CDK1.

Interestingly, IPA has recognized a massive number of regulated genes and were mainly involved in cell movement of cancer cell lines like CTNNB1, EGFR, ESR2, $H I F 1 A, R E L A$, and $W N T 3 A$ while some other genes were involved in cell death of various breast cancer cell lines like ECSIT, EWSR1, GPER1, KDM3A, MRTFB, and NCOA3. Moreover, DNA recombination, replication, and repair, cell cycle, survival, and death, and cellular development were among the highest regulated molecular and cellular functions after celastrol treatment.

\section{Discussion}

Natural products are an invaluable source of novel drug discovery because of their safety, potency, and lower cost. Due to deleterious side-effects of chemically synthesized therapeutics, especially anticancer; natural products are plausible alternatives and it is urgently needed to find safer natural remedies. ${ }^{35,47-49}$ Celastrol is a natural product obtained from Tripterygium wilfordii Hook F and was stated for its anticancer activities. Significantly, celastrol showed anticancer activity against various tumors as breast, prostate, and liver cancers, multiple myeloma, and glioma. ${ }^{21-28,35}$ Moreover, celastrol was used in treatments of chronic inflammation, autoimmune diseases, and neurodegenerative diseases. ${ }^{50}$ Despite there being some proposed mechanisms of celastrol's action in suppression of cancer, $^{35,50}$ its antitumor activity, especially in pancreatic cancer, has not been explored yet.

In the current analysis, the growth inhibitory effects of celastrol have been assessed on diverse pancreatic cancer cell lines with diverse proliferation stages and different resistance pattern. It was obvious that celastrol inhibited cellular growth of all tested cell lines compared to normal cells and the effect was cell line dependent where results showed that Miapaca-2 and BxPC-3 were the most affected cells while Capan- 2 and Panc-1 cells were the least affected cancer ones. Additionally, celastrol enhanced apoptosis in cancerous cells via activation of caspases 3/7 and an increasing amount of histone-linked DNA fragments released in cytoplasm. Moreover, the effect of celastrol on PGE2 (prostaglandin production) was also monitored where celastrol significantly inhibited PGE2 production in the BxPC-3 cell line (the highly COX-2 expressing cells). ${ }^{4,51}$ Furthermore, a comprehensive genome expression analysis was accomplished to identify the responsible genes for the growth inhibitory effects of celastrol, and its molecular targets in addition to the expected pathways that mediate its cellular effects in pancreatic cancer. 


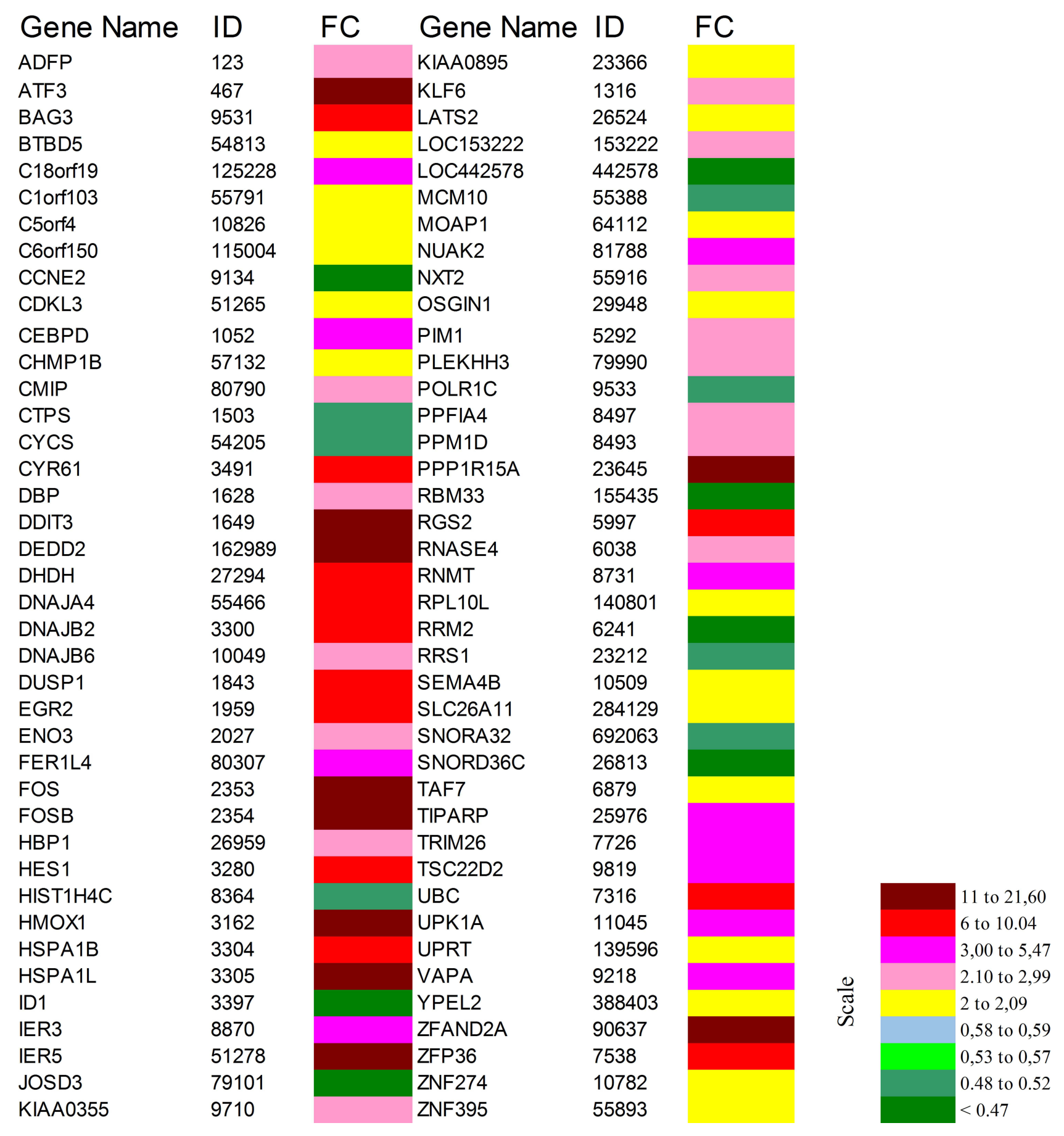

Figure 4 Top 80 pointedly up- or down-regulated genes in BxPC-3 cells after treatment with celastrol.

In the next few lines we will explain some genes upand down-regulated significantly in array results and how they helped us to explore the possible anticancer effect of celastrol on pancreatic cancer. These modulated genes after treatment have been previously reported for their correlations with biologically important pathways, cancer development, apoptosis, cell migration, metastasis, angiogenesis, and cell cycle and cyclin dependent pathways.
DNA-damage-inducible transcript 3 (DDIT3/ $G A D D 153)$ is an endoplasmic reticulum stress (ERS) related protein which was previously reported to have a noteworthy role in cancer. $^{52}$ Moreover, DDIT3 is known to play a crucial role in arresting apoptotic growth after DNA damage and after numerous stress circumstances, such as deprivation of nutrients and treatment with chemotherapeutic agents. ${ }^{5,53}$ Our array results 
Table 2 RT-PCR Verification of Microarrays Results for Some Selected Genes

\begin{tabular}{|l|l|c|c|}
\hline Official Gene Name & Gene Symbol & Fold Change (Microarray) & Fold Change (qRT-PCR) \\
\hline Ribonucleotide Reductase Regulatory Subunit M2 & RRM2 & -2.27 & -3.81 \\
\hline DNA-damage-inducible transcript 4 & DDIT4 & +14.06 & +8.22 \\
\hline Growth Arrest and DNA Damage Inducible Alpha & GADD45B & +3.26 & +2.17 \\
\hline Fos Proto-Oncogene & Fos & +21.9 & +11.52 \\
\hline
\end{tabular}

showed that DDIT3 encoding gene was among significantly up-regulated genes after treating of BxPC-3 cells with celastrol, and that could explain in part celastrol growth inhibitory effects.
Activating transcription factor 3 (ATF3) shows a significant role in modulation of stress and inflammatory responses. $A T F 3$ is an $A T F /$ cyclic AMP response elementbinding $(A T F / C R E B)$ transcription factors family member.

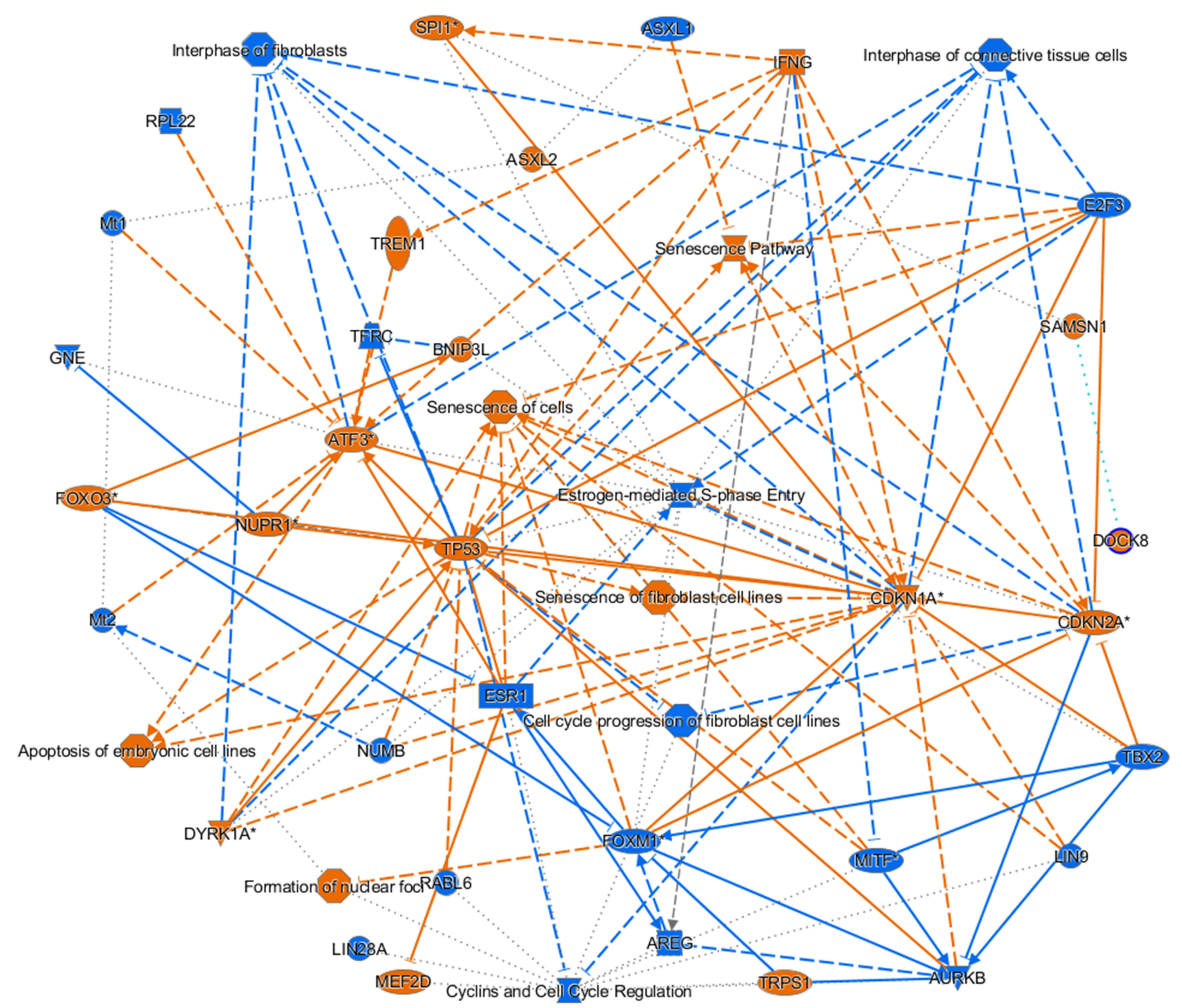

Figure 5 Graphical summary for pathways regulated after celastrol treatment. The topmost five regulated canonical pathways post-celastrol treatment were cyclins and cell cycle regulation, senescence pathway, cell cycle control and chromosomal regulation, cell cycle: GI/S checkpoint regulation, apoptosis of embryonic cell lines, estrogenmediated S-phase entry and prostatic cancer signaling. An asterisk $\left(^{*}\right)$ indicates that a given gene is represented in the microarray set with multiple probes. 


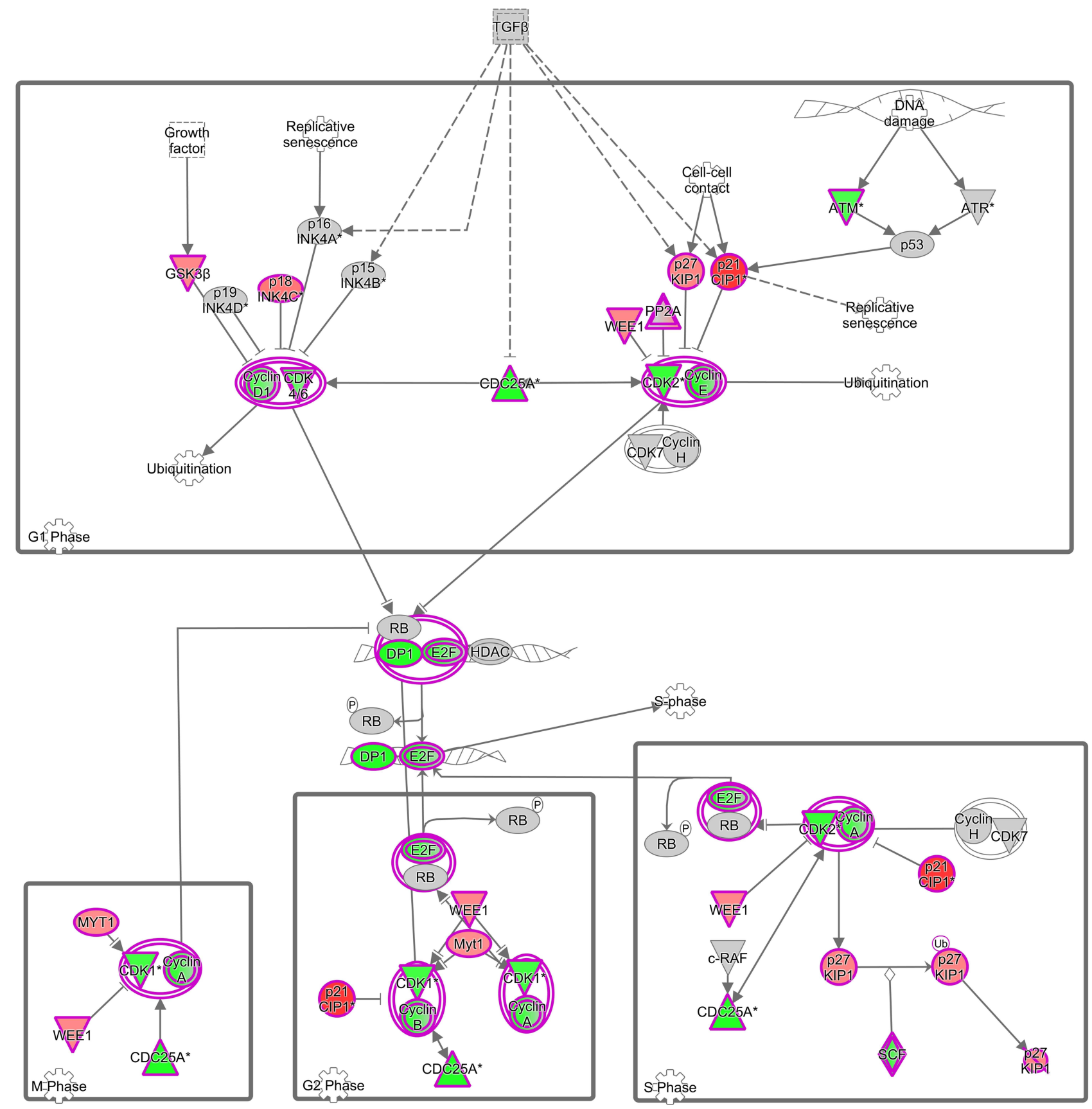

Figure 6 Cyclin and cell cycle regulation pathway after treatment of pancreatic cancer cells with celastrol. Example of upregulated genes (red) involved in cell cycle: $G / / S$ checkpoint regulation were P27KIPI, and PI8INK4C while among downregulated genes (green) involved in cell cycle: GI/S checkpoint regulation were Cyclin B, Cyclin A, and CDKI. An asterisk $(*)$ indicates that a given gene is represented in the microarray set with multiple probes.

Moreover, the role of $A T F 3$ has been shown to be critical for cancer progression by promoting or suppressing apoptosis and cell proliferation. ${ }^{54,55}$ However, the fluctuating role of $A T F 3$ on tumor progression, ATF3 notably exerts anticancer activity by regulation of CYR61. ${ }^{56}$ Recently, ATF3 was reported in hepatocellular carcinoma as a novel cancer suppressor gene. ${ }^{57}$ Our findings showed that $A T F 3$ was one of the significantly up-regulated genes after treating cancer cells with celastrol, which could participate in the celastrol's inhibitory effect on the growth of pancreatic cancer cells.

The rate-determining step in the synthesis of DNA is mediated by Ribonucleotide reductase (RRM). It was shown that RRM2 up-regulation is associated with gemcitabine anticancer resistance in pancreatic cancer patients. Furthermore, it was demonstrated that the efficacy of 
gemcitabine was enhanced by siRNA-based therapy of RRM2. ${ }^{5,58}$ Here, we report that RRM2 is down-regulated significantly in BxPC-3 cells after celastrol treatment. As far as we know, it is the first study that recognizes RRM2 as a candidate gene that can modulate the inhibitory effects of celastrol on pancreatic cancer cells growth.

Minichromosome maintenance (MCMs) family proteins play key roles in cellular replication and progression cycle. It was reported that the mRNA over-expression of MCM2, MCM4, and MCM10 genes were markedly enhanced lung adenocarcinoma, and over-expression of MCM2-5, MCM8, and MCM10 mRNA was linked to poor survival. $^{59-62}$ Our results indicated that MCM4 was significantly down-regulated after cancer cells treatment with celastrol. Accordingly, MCM4 down-regulation could be a proposed molecular mechanism that describes celastrol's inhibitory effect on pancreatic cancer cells.

In this study, we aimed to explore the exact celastrol's mechanisms of action on pancreatic cancer cells based on the most pointedly up- or down-regulated key player genes and for a comprehensive functional analysis of each single gene in pancreatic cancer. In this context and after confirmation of the anti-inflammatory, apoptotic, and anti-proliferative effects of celastrol on cells of pancreatic cancer; cDNA microarray was chosen as the best choice technique to screen its effect on thousands of genes alongside. The results of microarray pointed out the celastrol effects are mediated via multiple signaling pathways, and many genes were significantly involved in its inhibitory effect on cancer cells.

Finally, the outcome from the presented microarray results is considered a starting point to identify the exact molecular mechanisms of celastrol anticancer activities. This study gives precise insights about the prime targets of celastrol which may inspire us and others to further investigate celastrol and similar compounds for their pharmacological actions.

\section{Conclusion}

The current analysis obviously demonstrated that celastrol is a promising antitumor agent. Celastrol inhibited pancreatic cancer proliferation through affecting several signaling pathways. The celastrol's inhibitory effects on pancreatic cancer was basically attributed to DDIT3 and ATF3 activation and suppression of RRM2 and MCM4 genes. The microarray results and the expression analysis information that have been described here could be a starting point to recognize target genes and for a more thorough investigation of the functional role of each gene that describes the celastrol activity in prevention of cancer.

\section{Acknowledgment}

We are very thankful of Dr. Jörg Hoheisel, German Cancer Research Center (DKFZ), Heidelberg, Germany, for his continous support, and help. Additionally, the authors appreciate the efforts of Dr. Oliver Heil, Genomics and Proteomics Core Facility, DKFZ, Heidelberg, Germany, for his help in microarray data preparation and analysis.

\section{Disclosure}

The authors declare no conflicts of interest in this work.

\section{References}

1. Siegel RL, Miller KD, Jemal A. Cancer Statistics, 2017. CA Cancer J Clin. 2017;67(1):7-30. doi:10.3322/caac.21387

2. Siegel RL, Miller KD, Jemal A. Cancer statistics, 2016. CA Cancer J Clin. 2016;66(1):7-30.

3. Youns M, Fathy GM. Upregulation of extrinsic apoptotic pathway in curcumin-mediated antiproliferative effect on human pancreatic carcinogenesis. J Cell Biochem. 2013;114(12):2654-2665.

4. Torres Salazar A, Hoheisel J, Youns M, Wink M. Anti-inflammatory and anti-cancer activities of essential oils and their biological constituents. Int J Clin Pharmacol Ther. 2011;49(1):93-95.

5. Youns M, Efferth T, Reichling J, Fellenberg K, Bauer A, Hoheisel JD. Gene expression profiling identifies novel key players involved in the cytotoxic effect of Artesunate on pancreatic cancer cells. Biochem Pharmacol. 2009;78(3):273-283.

6. Gong Y, Zhang B, Liao Y, et al. Serum insulin-like growth factor axis and the risk of pancreatic cancer: systematic review and meta-analysis. Nutrients. 2017;9:4.

7. Tuli HS, Sandhu SS, Sharma AK. Pharmacological and therapeutic potential of Cordyceps with special reference to Cordycepin. 3 Biotech. 2014;4(1):1-12.

8. Youns M, Abdel Halim Hegazy W. The natural flavonoid fisetin inhibits cellular proliferation of hepatic, colorectal, and pancreatic cancer cells through modulation of multiple signaling pathways. PLoS One. 2017;12(1):e0169335.

9. Youns M, Fu YJ, Zu YG, et al. Sensitivity and resistance towards isoliquiritigenin, doxorubicin and methotrexate in T cell acute lymphoblastic leukaemia cell lines by pharmacogenomics. Naunyn Schmiedebergs Arch Pharmacol. 2010;382(3):221-234.

10. Saleh A, ElFayoumi HM, Youns M, Barakat W. Rutin and orlistat produce antitumor effects via antioxidant and apoptotic actions. Naunyn Schmiedebergs Arch Pharmacol. 2019;392(2):165-175.

11. Youns M, ElKhoely A, Kamel R. The growth inhibitory effect of gambogic acid on pancreatic cancer cells. Naunyn Schmiedebergs Arch Pharmacol. 2018;391(5):551-560.

12. Hegazy WAH. Hepatitis C virus pathogenesis: serum IL-33 level indicates liver damage. Afr J Microbiol Res. 2015;9(20):1386-1393.

13. Ashour ML, El-Readi MZ, Hamoud R, et al. Anti-infective and cytotoxic properties of Bupleurum marginatum. Chin Med. 2014;9 (1):4.

14. Chung MY, Lim TG, Lee KW. Molecular mechanisms of chemopreventive phytochemicals against gastroenterological cancer development. World J Gastroenterol. 2013;19(7):984-993.

15. Lee KW, Bode AM, Dong Z. Molecular targets of phytochemicals for cancer prevention. Nat Rev Cancer. 2011;11(3):211-218.

16. Ahmed MF, Youns M, Belal A. Design, synthesis, molecular docking and anti-breast cancer activity of novel quinazolinones targeting estrogen receptor alpha. Acta Pol Pharm. 2016;73(1):115-127. 
17. Kuete V, Krusche B, Youns M, et al. Cytotoxicity of some Cameroonian spices and selected medicinal plant extracts. J Ethnopharmacol. 2011;134(3):803-812.

18. Fatahala SS, Mohamed MS, Youns M, Abd-El Hameed RH. Synthesis and evaluation of cytotoxic activity of some pyrroles and fused pyrroles. Anticancer Agents Med Chem. 2017;17(7):1014-1025.

19. Pang X, Yi Z, Zhang J, et al. Celastrol suppresses angiogenesis-mediated tumor growth through inhibition of AKT/ mammalian target of rapamycin pathway. Cancer Res. 2010;70 (5):1951-1959.

20. Yadav VR, Sung B, Prasad S, et al. Celastrol suppresses invasion of colon and pancreatic cancer cells through the downregulation of expression of CXCR4 chemokine receptor. $J \mathrm{Mol}$ Med (Berl). 2010;88(12):1243-1253

21. Nakamichi K, Kitani H, Takayama-Ito M, Morimoto K, Kurane I, Saijo M. Celastrol suppresses morphological and transcriptional responses in microglial cells upon stimulation with double-stranded RNA. Int J Neurosci. 2010;120(4):252-257.

22. Zhu H, Liu XW, Cai TY, et al. Celastrol acts as a potent antimetastatic agent targeting betal integrin and inhibiting cell-extracellular matrix adhesion, in part via the p38 mitogen-activated protein kinase pathway. J Pharmacol Exp Ther. 2010;334(2):489-499.

23. Yu X, Tao W, Jiang F, Li C, Lin J, Liu C. Celastrol attenuates hypertension-induced inflammation and oxidative stress in vascular smooth muscle cells via induction of heme oxygenase-1. Am J Hypertens. 2010;23(8):895-903.

24. Wang XN, Wu Q, Yang X, Zhang LS, Wu YP, Lu C. Effects of Celastrol on growth inhibition of U937 leukemia cells through the regulation of the Notch1/NF-kappaB signaling pathway in vitro. Chin J Cancer. 2010;29(4):385-390.

25. Seo WY, Ju SM, Song HY, et al. Celastrol suppresses IFN-gammainduced ICAM-1 expression and subsequent monocyte adhesiveness via the induction of heme oxygenase- 1 in the HaCaT cells. Biochem Biophys Res Commun. 2010;398(1):140-145.

26. Salminen A, Lehtonen M, Paimela T, Kaarniranta K. Celastrol: molecular targets of Thunder God Vine. Biochem Biophys Res Commun. 2010;394(3):439-442.

27. Peng B, Xu L, Cao F, et al. HSP90 inhibitor, celastrol, arrests human monocytic leukemia cell U937 at G0/G1 in thiol-containing agents reversible way. Mol Cancer. 2010;9:79.

28. Jo H, Loison F, Hattori H, Silberstein LE, Yu H, Luo HR. Natural product Celastrol destabilizes tubulin heterodimer and facilitates mitotic cell death triggered by microtubule-targeting anti-cancer drugs. PLoS One. 2010;5(4):e10318.

29. Hou W, Liu B, Xu H. Celastrol: progresses in structure-modifications, structure-activity relationships, pharmacology and toxicology. Eur J Med Chem. 2020;189:112081.

30. Yang X, Wu F, Li L, et al. Celastrol alleviates metabolic disturbance in high-fat diet-induced obese mice through increasing energy expenditure by ameliorating metabolic inflammation. Phytother Res. 2021;35(1):297-310.

31. Yan CY, Ouyang SH, Wang X, et al. Celastrol ameliorates Propionibacterium acnes/LPS-induced liver damage and MSU-induced gouty arthritis via inhibiting K63 deubiquitination of NLRP3. Phytomedicine. 2021;80:153398.

32. Qiu N, Liu Y, Liu Q, et al. Celastrol nanoemulsion induces immunogenicity and downregulates PD-L1 to boost abscopal effect in melanoma therapy. Biomaterials. 2021;269:120604.

33. Chang TS, Wang TY, Chiang CM, et al. Biotransformation of celastrol to a novel, well-soluble, low-toxic and anti-oxidative celastrol-29-O-beta-glucoside by Bacillus glycosyltransferases. J Biosci Bioeng. 2021;131(2):176-182.

34. Abdelmoneem MA, Abd Elwakil MM, Khattab SN, et al. Lactoferrin-dual drug nanoconjugate: synergistic anti-tumor efficacy of docetaxel and the NF-kappaB inhibitor celastrol. Mater Sci Eng C Mater Biol Appl. 2021;118:111422.
35. Shi J, Li J, Xu Z, et al. Celastrol: a review of useful strategies overcoming its limitation in anticancer application. Front Pharmacol. 2020;11:558741.

36. Alhamdani MS, Youns M, Buchholz M, et al. Immunoassay-based proteome profiling of 24 pancreatic cancer cell lines. J Proteomics. 2012;75(12):3747-3759.

37. Qin Y, Meng $\mathrm{L}, \mathrm{Hu} \mathrm{C}$, et al. Gambogic acid inhibits the catalytic activity of human topoisomerase IIalpha by binding to its ATPase domain. Mol Cancer Ther. 2007;6(9):2429-2440.

38. Zhang Z, DuBois RN. Detection of differentially expressed genes in human colon carcinoma cells treated with a selective COX-2 inhibitor. Oncogene. 2001;20(33):4450-4456.

39. Strimpakos A, Saif MW, Syrigos KN. Pancreatic cancer: from molecular pathogenesis to targeted therapy. Cancer Metastasis Rev. 2008;27(3):495-522.

40. Ji S, Zhang B, Liu J, et al. ALDOA functions as an oncogene in the highly metastatic pancreatic cancer. Cancer Lett. 2016;374 (1):127-135

41. Galamb O, Spisak S, Sipos F, et al. Reversal of gene expression changes in the colorectal normal-adenoma pathway by NS398 selective COX2 inhibitor. Br J Cancer. 2010;102(4):765-773.

42. Mohamed MS, Youns MM, Ahmed NM. Synthesis, antimicrobial, antioxidant activities of novel 6-aryl-5-cyano thiouracil derivatives. Eur J Med Chem. 2013;69:591-600.

43. Mulyaningsih S, Youns M, El-Readi MZ, et al. Biological activity of the essential oil of Kadsura longipedunculata (Schisandraceae) and its major components. J Pharm Pharmacol. 2010;62(8):1037-1044.

44. de Souza Rocha Simonini P, Breiling A, Gupta N, et al. Epigenetically deregulated microRNA-375 is involved in a positive feedback loop with estrogen receptor alpha in breast cancer cells. Cancer Res. 2010;70(22):9175-9184.

45. Askoura M, Youns M, Halim Hegazy WA. Investigating the influence of iron on Campylobacter jejuni transcriptome in response to acid stress. Microb Pathog. 2020;138:103777.

46. Rothe K, Lin H, Lin KB, et al. The core autophagy protein ATG4B is a potential biomarker and therapeutic target in CML stem/progenitor cells. Blood. 2014;123(23):3622-3634.

47. Abbas HA, Hegazy WAH. Repurposing anti-diabetic drug "Sitagliptin" as a novel virulence attenuating agent in Serratia marcescens. PLoS One. 2020;15(4):e0231625.

48. Abd El-Hamid MI, et al. Promising antibiofilm agents: recent breakthrough against biofilm producing methicillin-resistant staphylococcus aureus. Antibiotics (Basel). 2020;9:10.

49. Hegazy WAH, Khayat MT, Ibrahim TS, et al. Repurposing anti-diabetic drugs to cripple quorum sensing in pseudomonas aeruginosa. Microorganisms. 2020;8:9.

50. Morita T. Celastrol: a new therapeutic potential of traditional Chinese medicine. Am J Hypertens. 2010;23(8):821.

51. Youns M, Efferth T, Hoheisel JD. Transcript profiling identifies novel key players mediating the growth inhibitory effect of NS-398 on human pancreatic cancer cells. Eur J Pharmacol. 2011;650 (1):170-177.

52. Bi M, Naczki C, Koritzinsky M, et al. ER stress-regulated translation increases tolerance to extreme hypoxia and promotes tumor growth. EMBO J. 2005;24(19):3470-3481.

53. Kim DG, You KR, Liu MJ, Choi YK, Won YS. GADD153-mediated anticancer effects of $\mathrm{N}$-(4-hydroxyphenyl)retinamide on human hepatoma cells. J Biol Chem. 2002;277(41):38930-38938.

54. Yang H, Park SH, Choi HJ, Moon Y. Epithelial cell survival by activating transcription factor 3 (ATF3) in response to chemical ribosome-inactivating stress. Biochem Pharmacol. 2009;77 (6):1105-1115.

55. Thompson MR, Xu D, Williams BR. ATF3 transcription factor and its emerging roles in immunity and cancer. $J$ Mol Med (Berl). 2009;87(11):1053-1060. 
56. Chen C, Ge C, Liu Z, et al. ATF3 inhibits the tumorigenesis and progression of hepatocellular carcinoma cells via upregulation of CYR61 expression. J Exp Clin Cancer Res. 2018;37(1):263.

57. Li L, Song S, Fang X, Cao D. Role of ATF3 as a prognostic biomarker and correlation of ATF3 expression with macrophage infiltration in hepatocellular carcinoma. BMC Med Genomics. 2021;14(1):8.

58. Goan YG, Zhou B, Hu E, Mi S, Yen Y. Overexpression of ribonucleotide reductase as a mechanism of resistance to 2,2-difluorodeoxycytidine in the human $\mathrm{KB}$ cancer cell line. Cancer Res. 1999;59(17):4204-4207.

59. Li S, Jiang Z, Li Y, Xu Y. Prognostic significance of minichromosome maintenance mRNA expression in human lung adenocarcinoma. Oncol Rep. 2019;42(6):2279-2292.
60. Kwok HF, Zhang SD, McCrudden CM, et al. Prognostic significance of minichromosome maintenance proteins in breast cancer. $\mathrm{Am}$ $J$ Cancer Res. 2015;5(1):52-71.

61. Hamamoto Y, Shomori K, Nosaka K, Haruki T, Teshima R, Ito H. Prognostic significance of Minichromosome maintenance protein 7 and Geminin expression in patients with 109 soft tissue sarcomas. Oncol Lett. 2010;1(4):703-709.

62. Nishihara K, Shomori K, Fujioka S, et al. Minichromosome maintenance protein 7 in colorectal cancer: implication of prognostic significance. Int J Oncol. 2008;33(2):245-251.

\section{Publish your work in this journal}

OncoTargets and Therapy is an international, peer-reviewed, open access journal focusing on the pathological basis of all cancers, potential targets for therapy and treatment protocols employed to improve the management of cancer patients. The journal also focuses on the impact of management programs and new therapeutic

Submit your manuscript here: https://www.dovepress.com/oncotargets-and-therapy-journal agents and protocols on patient perspectives such as quality of life, adherence and satisfaction. The manuscript management system is completely online and includes a very quick and fair peer-review system, which is all easy to use. Visit http://www.dovepress.com/ testimonials.php to read real quotes from published authors. 